\title{
Treatment of Metastatic Sweat Gland Carcinomas: Response in Two Cases
}

\section{Luo Liqiong ${ }^{1, *}$, Ding Bin ${ }^{2}$, Zeng Huiming ${ }^{2}$, Xiao Xiao ${ }^{1}$, Xiao Rong ${ }^{1}, \mathrm{Hu}_{\text {Lin }}{ }^{1}$}

${ }^{1}$ Department of Oncology, Tianyou Hospital Affiliated to Wuhan University of Science and Technology, Wuhan, 430064 China ${ }^{2}$ Clinical College Affiliated to Wuhan University of Science and Technology, Wuhan, 430064 China

${ }^{\star}$ Corresponding author: Luo Liqiong, Department of Oncology, Tianyou Hospital Affiliated to Wuhan University of Science and Technology, Wuhan, 430064 China; E-mail: luomengyun@hotmail.com

Received Date: May 17, 2019 Accepted Date: July 09, 2019 Published Date: July 10, 2019

Citation: Luo Liqiong (2019) Treatment of Metastatic Sweat Gland Carcinomas: Response in Two Cases. J Cancer Res Therap Oncol 7: 1-5.

\section{Introduction}

Sweat gland carcinomas are a rare group of tumors with the potential of destructing local tissue infiltration as well as regional and distant metastasis. Due to the limited availability of reference from the literature, the management of sweat gland carcinomas is both complex and cumbersome. Sweat gland carcinomas can be divided into eccrine and apocrine categories and occur primarily in adult patients, with a peak incidence during the fifth and sixth decades [1-2]. The majority of sweat gland carcinomas occur in the genital skin and perineum, followed by the trunk, head, neck and lower extremities. Regional and distant lymph node metastases frequently occur in a certain number of patients, but visceral metastases are seldom found. The metastasis sites mainly include lymph nodes, lungs and bone [3-5]. According to the available literature, radical surgical excision is the prior and standard treatment with the clearance of draining lymph nodes [6]. Some chemotherapeutic drugs, such as fluoropyrimidines, taxanes and cisplatin, have been reported to be active agents for metastatic sweat gland carcinomas [2,7-8]. However, the effect of adjuvant chemotherapy and radiotherapy remain elusive, and classical standards of diagnosis and therapy are still not clarified. Here, we report two cases of metastatic sweat gland carcinomas. In one case, the remission of nearly 6 months was achieved through chemotherapy of GP regimen; while in another case, the size of draining axillary lymph nodes was effectively controlled by oral administration of tamoxifen. 


\section{Case 1}

A 58-year-old patient, who presented fever and an apparent weight loss for two months duration, was registered in October 2014. Six months ago, the patient firstly noted a small single nodular lesion in the middle of his abdomen. Without regional lymph node dissection the patient accepted radiotherapy from the primary surgical bed (DT=54GY/27F). In August 2014, he developed intermittent fever of unknown origin and underwent a noticeable weight loss. The cause remained undetermined though various inspections had been conducted, and a variety of symptomatic treatments (without chemotherapy) seemed to be ineffective. In October 2014, examination of the axilla revealed bilaterally hard and mobile lymph nodes (the largest measuring $2.5 \times 2.5 \mathrm{~cm}$ and $3.5 \times 5.0 \mathrm{~cm}$ in diameter in the right and left axilla, respectively).CT scanning showed metastasis. After the patient was treated with two circles of chemotherapy (gemcitabine $1.0 \mathrm{~g} / \mathrm{m}^{2}$ on Day 1 and Day 8 and cisplatin $60 \mathrm{mg} / \mathrm{m}^{2}$ on Day1 every 21 days), the feeling of pain was significantly ameliorated with the disappearance of persistent fever. Examination of the axilla revealed obvious decreases in the size of lymph nodes (the largest measuring $0.5 \times 0.5 \mathrm{~cm}$ in diameter in the left axilla).CT results showed no significant changes in other metastatic lesions. The patient subsequently received four cycles of GP regimen with minimal clinical adverse reactions. In March 2015, multiple painful reddish raised nodular lesions appeared over the left chest and the left neck. Thereafter, the chemotherapy regimen was adjusted to docetaxel $75 \mathrm{mg} / \mathrm{m}^{2}$ andpirarubicin $50 \mathrm{mg} / \mathrm{m} 2$ on D1 every 21 days, which resulted in clinical response of bone marrow suppression. Thus, the chemotherapy regimen was abandoned. With the rapid progress of metastatic lesions for the following two months, the patient later died on May 15 due to complications A long survival (more than thirteen months) and a remarkable remission (nearly 6 months) were achieved.

The lesion was excised without draining lymph node dissection in June 2014.CT manifested enlarged bilateral axillary lymph nodes after surgery.

CT scanning showed swelling bilateral axillary lymph nodes, particularly on the left side, and metastasis to the liver, lungs, left adrenal and retroperitoneal lymph nodes.

The size of the swelling lymph nodes in bilateral axilla was obviously reduced.

The patient showed extensive cutaneous metastases over the left chest and the left neck after chemotherapy of GP regimen. After the chemotherapy of TA regimen, the conditions of the patient were recorded and observed by taking a photo every week. At first, the metastatic lesions on the skin gradually decreased in size and number. The chemotherapy was apparently effective at week 3 , but at week 4 , the metastatic lesions in the skin increased again, and at the same time showed darker color and harder base, indicating that the effect of the chemotherapy regimen was not lasting.

\section{Case 2}

A 71-year-old patient registered in the oncology in patient department presented enlargement of regional lymph

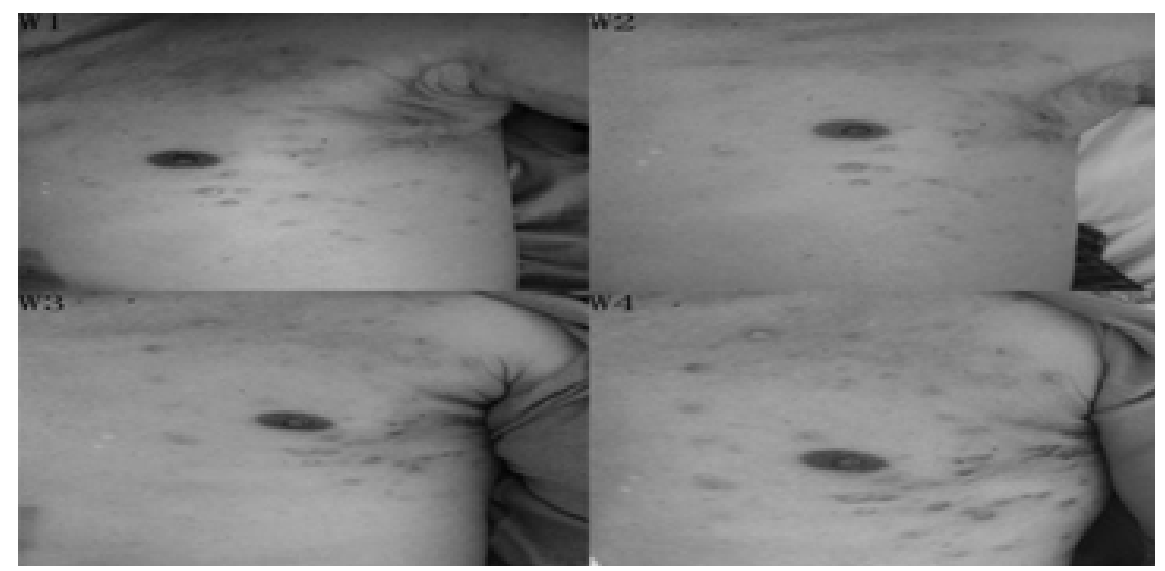

Figure: 1 Manifestation of metastatic sweat gland carcinoma after chemotherapy of TA regimen. 
nodes for the past one month. One month ago, he noticed that a small single nodular lesion located in the up-right abdomen for many years was gradually increasing in size with a rough surface. The lesion was locally excised without draining lymph node dissection in March 2018. He only accepted radiotherapy from the primary surgical bed due his old age and history of cerebrovascular diseases. However, he did not insist on radiotherapy after seven frequencies. In June 2018 physical examination revealed two hard and mobile nodules in the right axilla (about $2.0 \times 2.5$ $\mathrm{cm}$ and $1.7 \times 2.6 \mathrm{~cm})$.CT manifested metastasis. However, he again refused treatment of any regimen. In July 2018, physical examination revealed multiple grouped nodules (measuring 4.5 $\times 6.0 \mathrm{~cm}$ in diameter in the right axilla). CT manifested obvious progress of the metastatic lesions. Considering his special conditions, he finally accepted endocrino therapy of oral administration of tamoxifen with ER $(40 \%+)$. As a result, the size of the axillary lymph nodes did not show any further increase. The patient is still alive, and is under our follow-up observation.

\section{Histopathology}

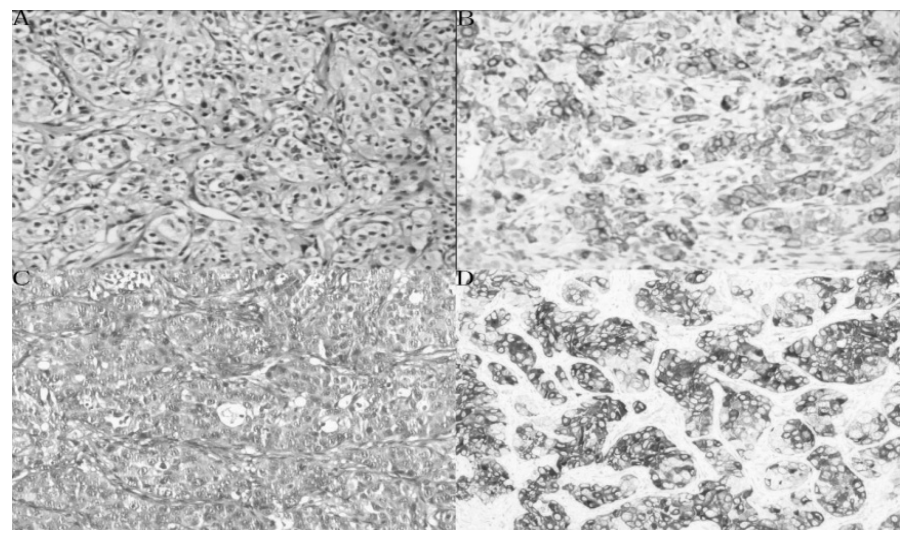

Figure 2 Histopathological and immunohistochemical images of two cases.

$\mathrm{A}$ and $\mathrm{C}$ represent the $\mathrm{H} \& \mathrm{E}$ staining images; $\mathrm{B}$ and $\mathrm{D}$ are the immunohistochemical images. B: $\operatorname{ER}(-), \operatorname{PR}(-), \operatorname{AR}(-)$, Her 2(-); D: ER(40\%+), PR(-), AR(-), Her2 (-). A and B represent the first case, $\mathrm{C}$ and $\mathrm{D}$ indicate the second case.

The hormonal levels of the two patients, the first case of a58-year-old patient and the second case of a71-year-old patient, were not tested after and before the treatment. Due to the poor conditions of the two country hospitals, it is a great pity that reports of histopathology for the two cases are not available.

\section{Discussion}

Sweat gland carcinomas are rare malignant tumors of the skin with a poor prognosis. Scalp is the most common site for the occurrence of sweat gland carcinomas, and the lesions may spread to the lymph nodes and the visceral organs with fatal results. Early identification and complete excision of sweat gland carcinomas provide the best chance of cure. Radical surgery of the primary lesions with wide regional lymph node dissection whenever possible is recommended for the initial and main treatment. Chemotherapy and/or radiotherapy have rather limited effects when employed for the palliative treatment of the advanced forms of the disease. Various cases have reported the use of a multitude of drugs in various sweat gland carcinomas, such as cyclophosphamide, doxorubicin, cisplatin, and taxanes (docetaxel and paclitaxel). However, only a very limited number of drugs are available for advanced sweat gland carcinomas, and treatments with these drugs only showed a low efficiency [2]. Besides, there has been no report about the application of gemcitabine in the treatment of metastatic sweat gland carcinomas.

Here, we report two cases of patients with sweat gland carcinomas in the abdomen, followed by axillary lymph metastasis and subsequent distant metastasis after treatment by local excision and adjuvant radiotherapy. Local excision and adjuvant radiotherapy were shown to be ineffective. Besides, all of the chemotherapy regimens failed to result in any significant clinical responses according to previous literature. Combined with the available literature, it can be concluded that wide local excision is a better treatment choice for these rare skin appendage tumors once they are localized, and adjuvant radiotherapy may be used to control the metastasis. Chemotherapy is considered as an option for more extensive lesions. In this report, the first-line chemotherapy of GP regimen did show great effects in the first case, indicating a relative curability of sweat gland carcinomas. However, the second-line chemotherapy of TA regimen did not show obvious effect.

There has been a report about the successful therapy of using adjuvant tamoxifen for metastatic sweat gland adenocarcinoma in an old female patient with $\operatorname{ER}(+)$ [9]. Here, we empirically applied tamoxifen citrate therapy, aiming to partly change the course of the disease. The therapy prevented the further increase in the size of the axillary lymph nodes, indicating the possible substantial therapeutic benefit of antiestrogen therapy [9-10]. Therefore, monitoring the hormone receptor expression in these neoplasms regularly will be of great significance, and the 
benefit of adjuvant anti-hormonal therapy in sweat gland adenocarcinoma should be further assessed.

In this paper, two cases of metastatic sweat gland carcinoma have been reported owing to the rarity and significant outcomes. A complete remission of six months duration was achieved for one patient, and a status of stable disease was achieved for another patient.

Due to the small number of reported cases, prognostic factors of sweat gland carcinomas, which possibly include lesion size, histological type, lymph node involvement and distant metastasis, are difficult to identify.
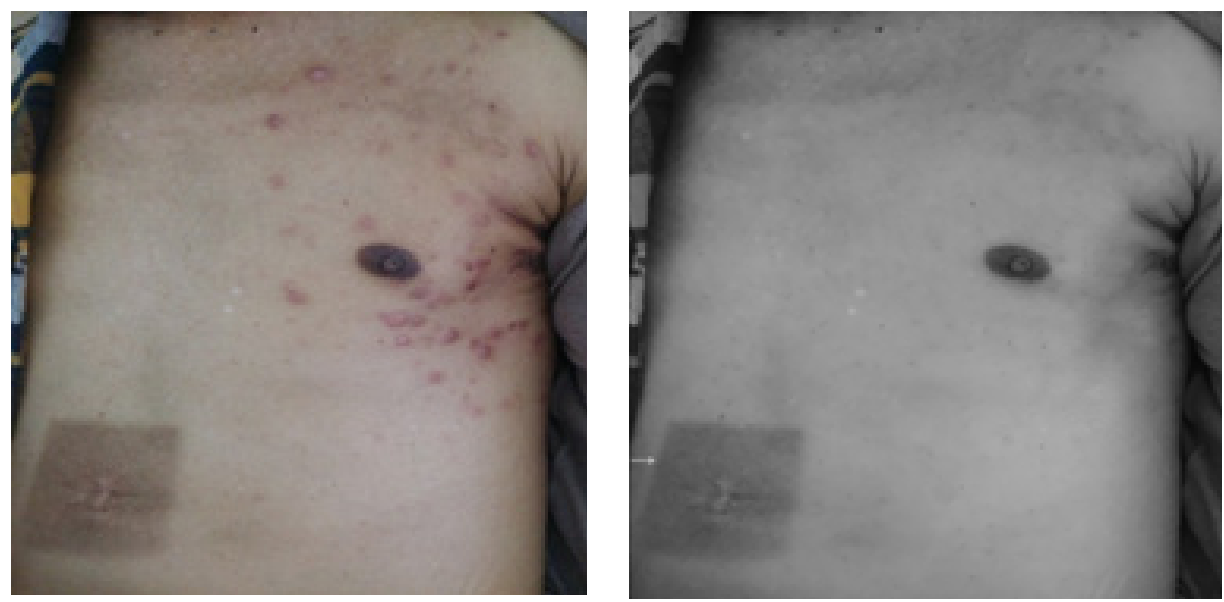

In October 2014,the pictures were not taken. However, the lesion of the patient after radiotherapy from the primary surgical bed remained unchanged as shown in the following pictures. Mean while, there was a small single nodular lesion in the middle of his abdomen.
Hence, larger and group-wide trials are needed to prospectively evaluate the application of chemotherapy and help the identification of these prognostic factors in advanced sweat gland carcinomas.

\section{Acknowledgements}

This research was financially supported by National Nature Science Foundation of China (81572495 and 81773264).

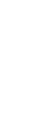




\section{References}

1. Doley B, Das AK, Das M (2001) Metastatic sweat gland carcinoma. J Assoc Physicians India 49:479-480.

2. Mezger J, Remberger K, Schalhorn A, Wohlrab A, Wilmanns W (1986) Treatment of metastatic sweat gland carcinoma by a four drug combination chemotherapy: response in two cases. Med Oncol Tumor Pharmacotherapy 3:29-34.

3. Chintamani1, Sharma R, Badran R, Singhal V, Saxena S, Bansal A (2003) Metastatic sweat gland adenocarcinoma: A clinico-pathological dilemma. World J Surg Oncol1:13.

4. Seong MK, Kim EK, Han K, Seol H, Kim HA, Noh WC M (2015) Primary apocrine sweat gland carcinomas of the axilla: a report of two cases and a review of the literature. World J. Surg Oncol 13:59.

5. Zahid R, Soofi ME, Elmalik H, Junejo K (2016) Primary apocrine carcinoma of the axilla in a male patient: a case report. Clin Case Rep 4:344-347.

6. Agha RA, Fowler AJ, Saeta A, Barai I, Rajmohan S, Orgill DP (2016) SCARE Group. The SCARE statement: consensusbased surgical case reportguidelines.Int J Surg 34:180-186.

7. Hollowell KL, Agle SC, Zervos EE, Fitzgerald TL (2012) Cutaneous apocrine adenocarcinoma: defining epidemiology, outcomes, and optimal therapy for a rare neoplasm. J Surg Oncol 105:415-419.

8. Joseph D, Dubashi B, Basu D, Srinivasan S, Jain A (2015) Response to taxane-based chemotherapy in metastatic eccrine porocarcinoma with extensive cutaneous involvement. Indian J Cancer 52:73-74.

9. Sridhar KS, Benedetto P, Otrakji CL, Charyulu KK (1989) Response of eccrine adenocarcinoma to tamoxifen.Cancer 64:366-370.

10. Schroder U, Dries V, Klussmann JP, Wittekindt C, Eckel HE (2004) Successful adjuvant tamoxifen therapy for estrogen receptor-positive metastasizing sweat gland adenocarcinoma: need for a clinical trial? Ann Otol Rhinol Laryngol 113:242-244.

Submit your manuscript to a JScholar journal and benefit from:

๑ Convenient online submission

- Rigorous peer review

ฯ Immediate publication on acceptance

ब Open access: articles freely available online

ब High visibility within the field

ब Better discount for your subsequent articles

Submit your manuscript at http://www.jscholaronline.org/submit-manuscript.php 